\title{
Upper Critical Field for Cobalt Oxide Superconductors
}

\author{
M. MierzeJewski ${ }^{a}$, M. MAŚKA $^{a}$ AND B. AndrzeJewski ${ }^{b}$ \\ ${ }^{a}$ Department of Theoretical Physics, Institute of Physics, Silesian University \\ Uniwersytecka 4, 40-007 Katowice, Poland \\ ${ }^{b}$ Institute of Molecular Physics, Smoluchowskiego 17, 60-179 Poznań, Poland
}

\begin{abstract}
Motivated by the recent discovery of the cobalt oxide superconductors, we calculate the temperature dependence of the upper critical field on a triangular lattice. Using the lattice version of the Gor'kov equations we investigate how the applied magnetic field affects singlet and triplet types of superconductivity. We show that in a wide range of model parameters not only Zeeman coupling, but also the diamagnetic pair breaking mechanism favors the triplet pairing. In the cobalt oxide superconductors the symmetry of the order parameter remains an open problem and both singlet and triplet superconductivity should be taken into account. We show that in such a case, an external magnetic field may induce a transition from singlet to triplet superconductivity. We discuss experimental results which may confirm this tempting hypothesis.
\end{abstract}

PACS numbers: 74.25.Op, 74.20.Rp

\section{Introduction}

Almost two decades after the discovery of high-temperature superconductivity a complete description of this phenomenon is missing. Extensive investigations of cuprate superconductors occurred to be insufficient to specify an appropriate microscopic model. Therefore, the recent discovery of superconductivity in a similar system, $\mathrm{Na}_{x} \mathrm{CoO}_{2} \cdot y \mathrm{H}_{2} \mathrm{O}$ [1], may be of crucial importance. The similarities between cuprate and cobalt oxide superconductors show up in dimensionality and strong electronic correlations. Co oxide becomes superconducting when the distance between $\mathrm{CoO}_{2}$ layers is enhanced by hydration. Above the superconducting transition temperature $\left(T_{\mathrm{c}}\right)$, the transport properties of the hydrated compound are of quasi two-dimensional character [2]. The presence of strong correlations 
may be responsible for a nonmonotonic doping dependence of $T_{\mathrm{c}}$, that resembles analogous dependence in cuprates [3]. However, the maximum of $T_{\mathrm{c}}$ takes place for doping that is approximately twice as large as the optimal doping in cuprates. In contradistinction to the optimally doped cuprates, the cobalt oxide superconductors are electron-doped systems. Other important difference is related to the lattice geometry. The $\mathrm{CoO}_{2}$ layers have a form of triangular lattice, where strong magnetic frustration occurs.

\section{Symmetry of the order parameter}

The pairing symmetry is currently the subject of intensive experimental and theoretical investigations. However, the existing experimental results often contradict each other $[4,5]$ and it remains unclear whether singlet or triplet pairing is responsible for superconductivity in $\mathrm{Na}_{x} \mathrm{CoO}_{2} \cdot y \mathrm{H}_{2} \mathrm{O}$. If the singlet pairing actually takes place, the resonating valence bond (RVB) state, constructed in terms of electron singlets, would be a straightforward explanation of superconductivity in the cobalt oxide $[6,7]$.

However, in the phase diagram proposed by Baskaran [6], in addition to RVB superconductivity, there is a region of triplet pairing that dominates at higher doping. It originates from the fact that in this regime phase fluctuations of the superconducting order parameter could be sufficiently strong to destroy the RVB state. Moreover, local density approximation (LDA) calculations suggest that the ground state of the parent system, $\mathrm{NaCo}_{2} \mathrm{O}_{4}$, may be ferromagnetic [8]. Recent density functional calculations performed for $\mathrm{Na}_{x} \mathrm{Co}_{2} \mathrm{O}_{4}$ predict an itinerant ferromagnetic state that, however, competes with a weaker antiferromagnetic instability [9]. Triplet superconductivity has also been postulated on the basis of symmetry considerations combined with analysis of experimental results [10].

Therefore, depending on the doping level both singlet and triplet pairings should seriously be taken into account. In particular, it is possible that singlet and triplet types of superconductivity compete with each other. In such a case not only large doping but also an external magnetic field may favor triplet pairing, due to the absence of the paramagnetic pair breaking mechanism in this state. In the following section we show that such a field-induced transition from singlet to triplet superconductivity should be visible in the temperature dependence of the upper critical field, $H_{\mathrm{c} 2}$. We recall also experimental results that support this hypothesis.

\section{Upper critical field}

We consider a triangular lattice in the presence of a uniform perpendicular magnetic field 


$$
\begin{aligned}
H= & \sum_{\langle i j\rangle \sigma} t_{i j} \mathrm{e}^{\mathrm{i} i_{i j}} c_{i \sigma}^{\dagger} c_{j \sigma}-\mu \sum_{i, \sigma} c_{i \sigma}^{\dagger} c_{i \sigma}-g \mu_{\mathrm{B}} H_{z} \sum_{i}\left(c_{i \uparrow}^{\dagger} c_{i \uparrow}-c_{i \downarrow}^{\dagger} c_{i \downarrow}\right) \\
& +V^{\mathrm{s}} \sum_{\langle i j\rangle}\left(\Delta_{i j} c_{i \uparrow}^{\dagger} c_{j \downarrow}^{\dagger}+\text { h.c. }\right)+V^{\mathrm{t}} \sum_{\langle i j\rangle} \sum_{\sigma, \nu=\uparrow \downarrow}\left(\Delta_{i j}^{\sigma \nu} c_{i \sigma}^{\dagger} c_{j \nu}^{\dagger}+\text { h.c. }\right) .
\end{aligned}
$$

$t_{i j}$ is the hopping integral in the absence of magnetic field and $\theta_{i j}$ is the Peierls phase factor, responsible for the diamagnetic pair breaking

$$
\theta_{i j}=\frac{2 \pi}{\Phi_{0}} \int_{i}^{j} \boldsymbol{A} \cdot \mathrm{d} \boldsymbol{l},
$$

where $\Phi_{0}=h c / e$ is the flux quantum. The third term in Eq. (1) is responsible for the paramagnetic pair breaking. Here, $g$ is the gyromagnetic ratio and $\mu_{\mathrm{B}}$ is the Bohr magneton. We have introduced the following order parameters:

$$
\Delta_{i j}=\left\langle c_{i \uparrow} c_{j \downarrow}-c_{i \downarrow} c_{j \uparrow}\right\rangle
$$

and

$$
\Delta_{i j}^{\uparrow \downarrow}=\left\langle c_{i \uparrow} c_{j \downarrow}+c_{i \downarrow} c_{j \uparrow}\right\rangle, \quad \Delta_{i j}^{\uparrow \uparrow}=\left\langle c_{i \uparrow} c_{j \uparrow}\right\rangle, \quad \Delta_{i j}^{\downarrow \downarrow}=\left\langle c_{i \downarrow} c_{j \downarrow}\right\rangle,
$$

which denote the pairing amplitudes in singlet and triplet channels, respectively.

In the following we do not specify the microscopic origin of the pairing interaction. In the simplest case, when the pairing originates from the nearest neighbor density-density attraction both the pairing potentials $V^{\mathrm{s}}$ and $V^{\mathrm{t}}$ are equal. We assume that $V^{\mathrm{s}}$ and $V^{\mathrm{t}}$ do not depend on the external magnetic field. The validity of this assumption depends on the nature of pairing potential and the strength of the magnetic field. For example, in the $t-J$ model [11] $J_{i j}(\boldsymbol{A})=4 t_{i j} \mathrm{e}^{\mathrm{i} \theta_{i j}} t_{j i} \mathrm{e}^{\mathrm{i}_{j i}} / U$ is strictly field independent, since the change of the phase generated when an electron hops from site $i$ to $j$ and back, cancels out. Such an assumption has been also partially justified on the basis of superconductivity driven by antiferromagnetic-spin-fluctuation [12].

In order to determine the temperature dependence of the upper critical field, $H_{\mathrm{c} 2}(T)$, we proceed the following steps: (i) applying a unitary transformation, that diagonalizes the kinetic part of the Hamiltonian, we introduce a new set of fermionic operators - in the case of the Landau gauge we end up with the Harper equation for the triangular lattice where the energy spectrum is known as the Hofstadter butterfly; (ii) we rewrite the Hamiltonian in terms of the new operators and (iii) construct the gap equation, that allows us to determine the critical temperature for a given magnetic field or, equivalently, $H_{\mathrm{c} 2}(T)$. For the details of this method we refer to Refs. [12, 13].

\section{Numerical results}

The temperature dependence of the upper critical field was obtained for singlet and triplet superconductivity. In the case of triplet pairing we investi- 
gated separately the paired states $|\downarrow \downarrow\rangle, \quad(1 / \sqrt{2})(|\uparrow \downarrow\rangle+|\downarrow \uparrow\rangle)$, and $|\uparrow \uparrow\rangle$. We refer to these states by the corresponding spin projection $S_{z}=-1,0,1$, respectively. These states are affected by the magnetic field in different ways. The singlet superconductivity as well as the triplet one with $S_{z}=0$ are significantly reduced by the Zeeman coupling. However, in the case of triplet equal-spin-pairing this mechanism is ineffective.

Figures 1 and 2 show numerical results obtained for $150 \times 150$ cluster with periodic boundary conditions along the $x$ axis and fixed boundary conditions in the perpendicular direction. Such a size of cluster is sufficient to obtain conver-

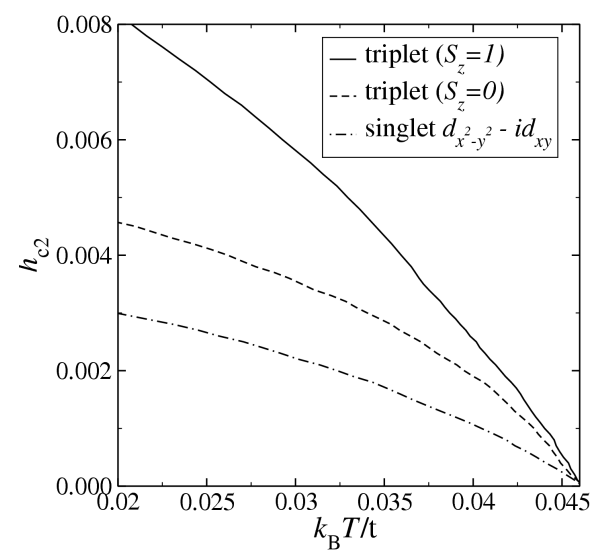

Fig. 1. Temperature dependence of the upper critical field obtained for occupation number $n=0.95$ and for $V^{\mathrm{s}}=0.55 t, V^{\mathrm{t}}=0.75 t$. We present a dimensionless reduced magnetic field, $h=2 \pi \Phi / \Phi_{0}$, where $\Phi_{0}$ is the flux quantum and $\Phi$ is the magnetic flux through the lattice cell ( $\Phi=H a^{2} \sqrt{3} / 2$, where $a$ is the lattice constant).

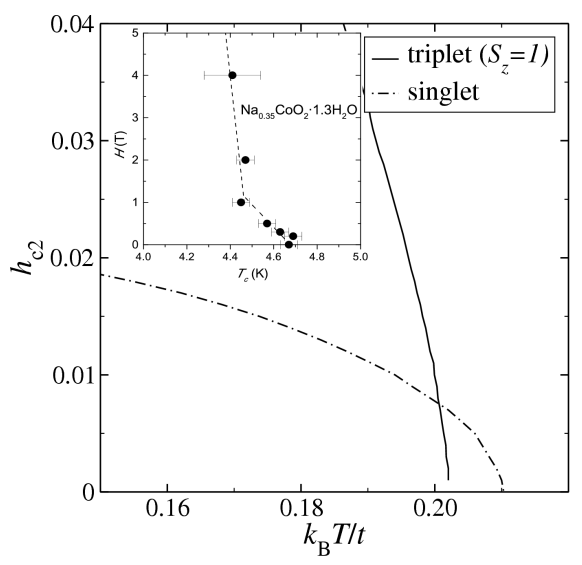

Fig. 2. $h_{\mathrm{c} 2}(T)$ for $n=0.67$ and $V^{\mathrm{s}}=V^{\mathrm{t}}=0.7 t$ (see Fig. 1 for the notation). The inset shows experimental results taken from Ref. [14]. 
gent results [12]. We have considered various occupation numbers and magnitudes of the pairing potentials. We have found that triplet superconductivity is characterized by higher upper critical field than the singlet one, provided $T_{\text {c }}$ 's for both the types of pairing are of comparable magnitudes. This result originates not only from the Zeeman coupling that favors equal-spin-pairing. Figure 1 shows that also diamagnetic pair breaking is less effective for the triplet superconductivity. In the presence of both the pair breaking mechanisms, the triplet superconductivity with $S_{z}= \pm 1$ is characterized by the highest value of $H_{\mathrm{c} 2}$, whereas the lowest one corresponds to the singlet superconductivity. The most interesting case occurs when the transition temperature for the triplet superconductivity is slightly less than $T_{\mathrm{c}}$ in the singlet channel. Then, as depicted in Fig. 2, sufficiently strong magnetic field leads to a transition from singlet to triplet superconductivity that shows up in a change of the slope of $H_{\mathrm{c} 2}(T)$. Within the scenario proposed by Baskaran [6] this transition should occur for moderate doping, i.e. close to the boundary between the singlet and triplet phases.

\section{Discussion and conclusions}

The change of slope of $H_{\mathrm{c} 2}(T)$ in the cobalt oxide superconductors has been reported for the first time in Ref. [14]. Such a bend in a weak field regime is visible also in magnetization [15] and resistivity measurements (see Fig. 4a in Ref. [16]). In order to investigate this feature in more details we have carried out precise measurements of the upper critical field $\left(H_{\mathrm{c} 2}\right)$ in a weak field regime. Figure 3 shows the results. For $1 \mathrm{~T}<H<3 \mathrm{~T}$ the experimental data can be fitted very well by a linear function. However, such a fit deviates from experimental points for weaker magnetic field. Since the temperatures presented in Fig. 3 are close to $T_{\mathrm{c}}$ one might expect that the standard Ginzburg-Landau theory gives accurate results and, therefore, temperature dependence of $H_{\mathrm{c} 2}$ should be linear. This

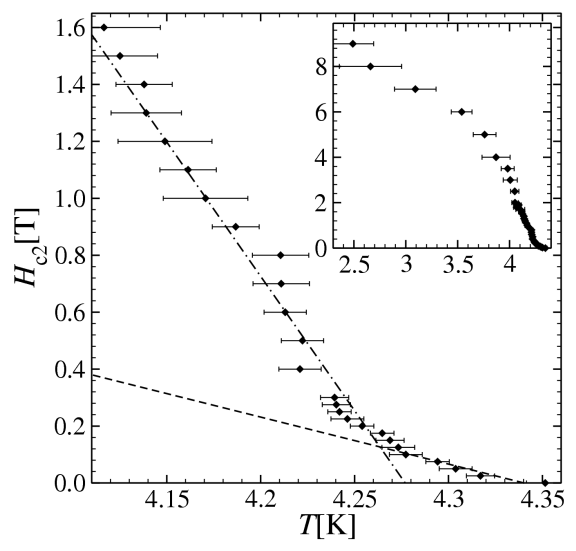

Fig. 3. $H_{\mathrm{c} 2}(T)$ determined experimentally for $\mathrm{Na}_{0.3} \mathrm{CoO}_{2} \cdot 1.3 \mathrm{H}_{2} \mathrm{O}$. For the details see Ref. [17]. 
discrepancy can be explained in terms of field induced phase transition between various superconducting order parameters. Singlet and triplet order parameters are the most natural candidates because they are characterized by different slopes of $H_{\mathrm{c} 2}(T)$, as we have shown in the previous section. Moreover, $H_{\mathrm{c} 2}(T)$ exceeds the Clogston-Chandrasekhar $(\mathrm{CC})$ limit $(\approx 8 \mathrm{~T}$ in the present case) already for $T \approx 0.6 T_{\mathrm{c}}$. This speaks in favor of triplet superconductivity in the strong field regime. On the other hand, $H_{\mathrm{c} 2}(0)$ estimated from the linear fit to the low field data does not exceed $\mathrm{CC}$ limit and superconductivity in this regime may originate from the singlet pairing. Here, there exists also other possibility: $H_{\mathrm{c} 2}(T)$ for the field less than approximately $0.9 \mathrm{~T}$ can be fitted by a concave curve. Similarity between $\mathrm{Na}_{x} \mathrm{CoO}_{2} \cdot y \mathrm{H}_{2} \mathrm{O}$ and high- $T_{\mathrm{c}}$ superconductors may suggest a common mechanism that leads to the positive curvature of $H_{\mathrm{c} 2}(T)$. This also speaks in favor of a singlet pairing in the low field regime.

\section{References}

[1] K. Takada, H. Sakurai, E. Takayama-Muromachi, F. Izumi, R.A. Dilanian, T. Sasaki, Nature 422, 53 (2003).

[2] R. Jin, B.C. Sales, P. Khalifah, D. Mandrus, cond-mat/0306066.

[3] R.E. Schaak, T. Klimczuk, M.L. Foo, R.J. Cava, Nature 424, 527 (2003).

[4] T. Waki, C. Michioka, M. Kato, K. Yoshimura, K. Takada, H. Sakurai, E. Takayama-Muromachi, T. Sasaki, cond-mat/0306026; Y. Kobayashi, M. Yokoi, M. Sato, cond-mat/0306264.

[5] Y. Kobayashi, M. Yokoi, M. Sato, J. Phys. Soc. Jpn. 72, 2161 (2003); T. Fujimoto, G.-Q. Zheng, Y. Kitaoka, R.L. Meng, J. Cmaidalka, C.W. Chu, Phys. Rev. Lett. 92, 047004 (2004); K. Ishida, Y. Ihara, Y. Maeno, C. Michioka, M. Kato, K. Yoshimura, K. Takada, T. Sasaki, H. Sakurai, E. Takayama-Muromachi, J. Phys. Soc. Jpn. 72, 3041 (2003).

[6] G. Baskaran, Phys. Rev. Lett. 91, 097003 (2003).

[7] T. Koretsune, M. Ogata, Phys. Rev. Lett. 89, 116401 (2002).

[8] D.J. Singh, Phys. Rev. B 61, 13397 (2000).

[9] D.J. Singh, Phys. Rev. B 68, 020503 (2003).

[10] A. Tanaka, X. Hu, Phys. Rev. Lett. 91, 257006 (2003).

[11] K.A. Chao, J. Spałek, A.M. Oleś, Phys. Rev. B 18, 3453 (1978).

[12] M. Maśka, M. Mierzejewski, Phys. Rev B 64, 064501 (2001).

[13] M. Mierzejewski, M.M. Maśka, Phys. Rev. B 60, 6300 (1999).

[14] H.D. Yang, J.Y. Lin, C.P. Sun, Y.C. Kang, K. Takada, T. Sasaki, H. Sakurai, E. Takayama-Muromachi, cond-mat/0308031.

[15] H. Sakurai, K. Takada, S. Yoshii, T. Sasaki, K. Kindo, E. Takayama-Muromachi, Phys. Rev. B 68, 132507 (2003).

[16] T. Sasaki, P. Badica, N. Yoneyama, K. Yamada, K. Togano, N. Kobayashi, J. Phys. Soc. Jpn. 73, 1131 (2004).

[17] M. Maśka, M. Mierzejewski, B. Andrzejewski, M.L. Foo, R.J. Cava, T. Klimczuk, Phys. Rev. B, 2004, in press. 